\title{
Cognitive impairment in ALS patients and validation of the Spanish version of the ALS-CBS test
}

\author{
JANINA TURON-SANS ${ }^{1}$, JORDI GASCON-BAYARRI ${ }^{1}$, RAMON REÑÉ ${ }^{1}$, IMMA RICO ${ }^{1}$, \\ CRISTINA GÁMEZ ${ }^{2}$, ANDRES PAIPA ${ }^{1} \&$ MONICA POVEDANO $^{1}$ \\ ${ }^{1}$ Department of Neurology, Bellvitge University Hospital, L'Hospitalet de Llobregat and ${ }^{2}$ IDI-PET-Bellvitge \\ University Hospital-IDIBELL, L'Hospitalet de Llobregat, Spain
}

\begin{abstract}
Our aim was to develop and validate the Spanish version of the Amyotrophic Lateral Sclerosis Cognitive Behavioural Screen (ALS-CBS) and investigate behavioural/cognitive impairment in our ALS patients. We enrolled 50 patients with definite or probable ALS, evaluated by the Motor Neuron Disease Unit (using El Escorial criteria) and Dementia Unit, and assessed with the Spanish ALS-CBS. The patients' cognitive/behavioural status was classified according to current criteria. Patients were classified into each diagnostic category: ALS-no impairment, 36\%; ALS-mild cognitive impairment, 34\%; ALS-mild behavioural impairment, 6\%; ALS-mild cognitive/behavioural impairment, 12\%; ALS-frontotemporal dementia, $12 \%$. Cognitive impairment was more common in bulbar $(90.9 \%)$ than spinal $(48.7 \%)$ forms $(p<0.012)$. The Spanish ALS-CBS was validated. Performance to differentiate normal vs. impaired individuals was: 1) cognition (cut-off 15; AUC, $84.7 \%$ ): sensitivity $86.2 \%$, specificity $62 \%$, positive predictive value $75.8 \%$, negative predictive value $76.5 \%$; 2 ) behaviour (cut-off 36; AUC, 83.3\%): sensitivity $93.3 \%$, specificity $74.3 \%$, positive predictive value $61 \%$, negative predictive value 96.3\%. Performance to differentiate between patients with and without dementia: 1) cognition (cut-off 8; AUC, 87.3\%): sensitivity $83.3 \%$, specificity $75 \%$, positive predictive value $31.3 \%$, negative predictive value $97.1 \%$; 2) behaviour (cut-off 35; AUC, 80.9\%): sensitivity $83.3 \%$, specificity $69 \%$, positive predictive value $25 \%$, negative predictive value $96.7 \%$. In conclusion, cognitive impairment is common in ALS patients, particularly in bulbar forms. The Spanish version of the ALS-CBS is useful for screening cognitive/behavioural impairment in this population.
\end{abstract}

Key words: Amyotrophic lateral sclerosis, frontotemporal dementia, ALS-CBS, cognitive impairment

\section{Introduction}

Amyotrophic lateral sclerosis (ALS) was formerly considered a disease mainly affecting the motor system, with denial bias regarding cognitive and behavioural symptoms. Motor symptoms and their impact on the patients' daily life have further hindered recognition of cognitive/behavioural manifestations (1). Over recent decades, an increasing number of publications have focused on these aspects, with emergence of diagnostic criteria for frontotemporal dementia (FTD) (2) and mild cognitive impairment (3), both of which can be present in ALS patients. It is now known that $7 \%-41 \%$ of ALS patients have FTD and $10 \%-70 \%$ have mild cognitive/behavioural impairment, depending on the series and diagnostic criteria used (4).
There are no epidemiological studies on the prevalence and incidence of cognitive/behavioural impairment in ALS patients in Spain, despite reports advocating routine cognitive/behavioural assessment in ALS hospital units (4). The reference standard for this purpose is neuropsychological examination, which is costly, time-consuming, and not always applicable to this population. Because of these limitations, several tools have been designed to estimate the cognitive/behavioural state of these patients. One such instrument is the ALS Cognitive Behavioural Screen (ALS-CBS) (5).

The aims of this study were to validate the Spanish version of the ALS-CBS and determine the percentage of ALS patients presenting cognitive/behavioural impairment in our setting.

Correspondence: J. Gascon Bayarri, Servei de Neurologia, Hospital Universitari de Bellvitge, Avda. de la Feixa Llarga s/n, 08907-L'Hospitalet de Llobregat, Barcelona, Spain. Fax: 34 932607365. E-mail: jordigneuro@bellvitgehospital.cat;32535jgb@comb.cat 


\section{Patients and methods}

The study included consecutive new patients of any age and either gender attending the Motor Neuron Disease Unit of our hospital. Patients who met the revised El Escorial diagnostic criteria (6) for definite or probable ALS and were accompanied by a valid caregiver in the opinion of the attending physician were considered candidates. Patients who could not be evaluated because of their clinical condition or sensory deficits were excluded. Patients gave informed consent for participation.

The initial visit to investigate suspected motor neuron disease included detailed clinical history taking, neurological examination, laboratory analyses (thyroid function, cobalamin, folate, creatine kinase, urate, ferritin, protein and lipid profiles, syphilis serology) and genetic sequencing. In addition, patients underwent assessment with the ALS Functional Rating Scale-Revised (ALSFRS-R) (7) and forced vital capacity (FVC) testing. Electromyography study and lumbar puncture were performed, and cranial/cervical magnetic resonance (MR) imaging and 18-FDG positron emission tomography (PET) of the brain were requested. The neuroimaging results will be reported in another, related paper.

Patients who met the inclusion criteria and had no criteria for exclusion were then assessed by two neurologists with broad experience evaluating cognitive/behavioural impairment (Dementia Unit). The visit included history taking, neurological examination, the Folstein MMSE test (8), and the Spanish version of the ALS-CBS, which was always administered after the neurological diagnosis. Findings from complementary tests were also evaluated. Clinical criteria were used to conclude the diagnosis, which was based on the recent consensus criteria for diagnosing cognitive/behavioural impairment in ALS patients (4) and the Neary criteria for FTD (2). Six diagnostic categories were established:

- ALS with no cognitive/behavioural impairment

- ALS with mild cognitive impairment (ALSci)

- ALS with mild behavioural impairment (ALSbi)

- ALS with mild cognitive and behavioural impairment (ALScibi)

- ALS with frontotemporal dementia (ALS-FTD)

- ALS with another type of dementia

Within 30 days after the neurological examination, patients were seen by a neuropsychologist blinded to the neurologist's findings. Patients were tested with a neuropsychological battery created for the study to classify them according to the Neary criteria for FTD diagnoses (2) and the consensus criteria for mild impairment diagnoses (4). To establish mild cognitive impairment, the patient's performance had to be at or below the 5th percentile compared to age- and education-matched norms on at least two different cognitive tests, and could not be related to the premorbid intellectual level, bulbar dysfunction, motor weakness, or to neurological, systemic, pharmacological, or severe psychiatric comorbidities. Mild behavioural impairment was diagnosed on the presence of two non-overlapping behavioural abnormalities supported by at least two sources from among a patient interview/observation, caregiver report, or structured questionnaire/interview, and after having ruled out a psychiatric condition, psychological reaction, premorbid personality disorder, or a pseudobulbar affect. In all patients studied, the assessment took into account normative values according to age and educational level for the Spanish population.

The battery included several tests. Executive function was investigated with the Category Fluency (animals) and Letter Fluency (using P, M, and R) tasks $(9,10)$ to assess fluency; the Stroop Color and Word Test (11) and Trail Making Test (12) to evaluate inhibition and mental flexibility, the Wisconsin Card Sorting Test (13) and Tower of London Test (14) to study abstraction and problem solving, the Similarities Test (15) to check verbal reasoning, and the WMS-III Digits subtest (16) to evaluate selective attention and working memory.

Language assessment used the Boston Naming Test (17), and semantic knowledge was evaluated with the Complex Ideation/Commands Test (9) and Pyramids and Palm Trees Test (18).

Psychomotor speed was assessed with the WAISIII Symbol Search (15), memory and verbal learning with the Rey Auditory Verbal Learning Test (19), visual memory with the WMS-III Faces and Family Pictures subtests (20), and visuospatial ability with the Judgement of Line Orientation Test $(21,22)$. Premorbid IQ was estimated with the WAIS-III Vocabulary subtest (15).

Psychopathological and neuropsychiatric symptoms were evaluated with the Beck Depression Inventory (23), Neuropsychiatric Inventory (24,25), Apathy Scale (26), and Frontal Behavioural Inventory (27).

The ALS-CBS instrument has two parts, a cognitive section and a behavioural questionnaire. The cognitive section comprises four subscales yielding a total score of 20, with lower scores indicating greater impairment. Responses are provided verbally or in writing. Scoring is based on a combination of the scores for each item and the number of errors made, in keeping with the rules of the original version. The behavioural questionnaire includes 15 caregiver-rated items that assess changes noted in the patient since disease onset. Items are scored from 0 (a large change) to 3 (no change) and the total ranges from 0 to 45 .

A fully bilingual professional translator was engaged to translate the English text into Spanish. The letter F, used in the English version as the guide for evaluating verbal fluency, was preserved in the 
Spanish version, as the percentage of words starting with $\mathrm{F}$ is similar in the two languages. Thus, the same cut-off could be used in the Spanish version as in the original. A second professional translator performed a back translation into English of the Spanish text to confirm that the original and the back translation were equivalent.

\section{Data collection}

Independent variables collected from patients and caregivers were recorded in an ad hoc encrypted database, created using the Bento program for iPad. The information included imaging findings, the diagnostic categories ascribed to patients by clinicians, the neuropsychologist's evaluation, and the ALS-CBS scores.

\section{Statistics}

Statistical analyses were carried out with SPSS, version 15.0. The mean, range, and standard deviation were calculated for descriptive variables, MMSE test scores, and ALS-CBS scores. The percentages of patients in each diagnostic category classified according to the two assessments (neurologist/neuropsychologist) were also calculated. Between-group comparisons were performed using a Student's $t$-test for means, a $\chi^{2}$ test for percentages, and a Fisher's $\mathrm{F}$ test for standard deviations. A $p$ value of $<0.05$ was considered significant. Variables showing significant differences were included in a logistic regression model.

The sensitivity, specificity, and positive and negative predictive values of the Spanish ALS-CBS were calculated relative to the reference standard neuropsychological evaluation. Optimal cut-off values for diagnosing ALS-related cognitive/behavioural impairment and FTD were also established.

\section{Results}

Between 1 October 2012 and 1 October 2013, 78 patients who met the inclusion criteria and had no criteria for exclusion were enrolled. Twenty-six patients were unable to complete the neuropsychological study. One patient (ALSci) was excluded due to depression (BDI score was 23, and the investigator judged that the clinical-neurological evaluation would be affected) and another due to previous head trauma. Of the 50 patients completing the study, $64 \%$ were males and mean age was 62.3 years (range $36-86 ; 32 \%<60$ years, $32 \%>70$ years). Almost half $(48 \%)$ had less than eight years of schooling. A family history of dementia was recorded in eight patients (seven Alzheimer's disease), motor neuron disease in two, and Parkinson's disease in four. Mean disease duration since symptoms onset was 18 months (range 3-68; 40\%<12 months, 16\%>24 months) (Table I). In total, $76 \%$ of patients had spinal-onset ALS and 22\% bulbar onset (dysarthria in $90 \%$ ). At the time of the study, $58 \%$ of patients had bulbar symptoms.

Based on the neuropsychological assessment, $12 \%$ of patients had FTD. Some degree of frontotemporal cognitive/behavioural impairment (including dementia) was seen in $64 \%$ of patients. Also based on the neuropsychological assessment, cognitive impairment (ALSci + ALScibi + ALSFTD) was diagnosed in 58\% and behavioural disorder (ALSbi + ALS-FTD) in 30\% of patients. The most commonly affected cognitive domains were executive function (60\%), verbal or visual memory $(35 \%)$, and language $(12 \%)$. When verbal fluency $(31 \%)$ was removed from executive function, this domain was affected in $29 \%$ of cases and language in $15 \%$. The most common psychobehavioural symptoms were irritability (40\%), impatience $(30 \%)$, depression (24\%), apathy (12\%), and disinhibition (10\%). Emotional lability was observed in $58 \%$ of cases.

Mean MMSE score was 27 points (range 16-30). Mean ALS-CBS scores were 12 points (range 1-19) for the cognitive section and 34 points $(0-45)$ for the behavioural. The percentages of patients ascribed to each diagnostic category based on the clinicians' and neuropsychologist's evaluation, respectively, were as follows: normal 30\%, 36\%; ALSci $34 \%$, 34\%; ALSbi 8\%, 6\%; ALScibi 16\%,

Table I. Patient demographic characteristics.

\begin{tabular}{|c|c|c|c|c|c|c|c|}
\hline Age, years & $\begin{array}{c}\text { Females } \\
n(\%)\end{array}$ & $\begin{array}{c}\text { Males } n \\
(\%)\end{array}$ & $\begin{array}{l}\text { Total } \\
(\%)\end{array}$ & Education, years & $\begin{array}{c}\text { Females } \\
n(\%)\end{array}$ & $\begin{array}{l}\text { Males } \\
n(\%)\end{array}$ & $\begin{array}{l}\text { Total } \\
n(\%)\end{array}$ \\
\hline$<60$ & $6(37.5)$ & $10(62.5)$ & $16(32)$ & None or $<8$ & $7(29.2)$ & $17(70.8)$ & $24(48)$ \\
\hline $60-69$ & $8(44.4)$ & $10(55.6)$ & $18(36)$ & $8-14$ & $7(46.7)$ & $8(53.3)$ & $15(30)$ \\
\hline$\geq 70$ & $4(25)$ & $12(75)$ & $16(32)$ & $\geq 15$ & $4(36.4)$ & $7(63.6)$ & $11(22)$ \\
\hline Total & $18(36)$ & $32(64)$ & $50(100)$ & Total & $18(36)$ & $32(64)$ & $50(100)$ \\
\hline $\begin{array}{l}\text { Time since ALS } \\
\text { onset, months }\end{array}$ & $\begin{array}{l}\text { Females } \\
n(\%)\end{array}$ & $\begin{array}{l}\text { Males } \\
n(\%)\end{array}$ & $\begin{array}{l}\text { Total } \\
n(\%)\end{array}$ & $\begin{array}{l}\text { Time since ALS } \\
\text { onset, months }\end{array}$ & $\begin{array}{l}\text { Bulbar } \\
n(\%)\end{array}$ & $\begin{array}{l}\text { Spinal } \\
n(\%)\end{array}$ & $\begin{array}{l}\text { Total } \\
n(\%)\end{array}$ \\
\hline$<12$ & $9(45)$ & $11(55)$ & $20(40)$ & $<12$ & $3(15)$ & $17(85)$ & $20(40)$ \\
\hline $12-23$ & $8(36.4)$ & $14(63.6)$ & $22(44)$ & $12-23$ & $8(36.4)$ & $14(63.6)$ & $22(44)$ \\
\hline$\geq 24$ & $1(12.5)$ & $7(87.5)$ & $8(16)$ & $\geq 24$ & $1(12.5)$ & $7(87.5)$ & $8(16)$ \\
\hline Total & $18(36)$ & $32(64)$ & $50(100)$ & Total & $11(22)$ & $38(76)$ & $50(100)$ \\
\hline
\end{tabular}

ALS: amyotrophic lateral sclerosis. 
$12 \%$; and ALS-FTD 12\%, $12 \%$; two patients in this last category were diagnosed with ALS-FTD plus Parkinson's disease. Overall agreement between the two evaluations was $90 \%$, and agreement for ALS-FTD was $100 \%$. No significant differences were found between those who underwent the complete study and those who did not (Table II).

The clinical-neurological evaluation results and ALS-CBS scores were compared with the reference standard (neuropsychological evaluation) to validate the Spanish version of the ALS-CBS. To detect significant differences in the variables studied, the sample was divided, and two separate analyses were performed: the first included ALS patients with normal cognitive/behavioural status versus those

Table II. Demographic differences between patients who completed both the neurological and neuropsychological evaluations $(n=50)$ and those who did not complete the neuropsychological evaluation $(n=28)$.

\begin{tabular}{|c|c|c|c|}
\hline & $\begin{array}{l}\text { Complete } \\
\text { evaluation } \\
(n=50) \\
\end{array}$ & $\begin{array}{c}\text { Only neurological } \\
\text { evaluation } \\
(n=28)\end{array}$ & $p$ \\
\hline Men & $64.0 \%$ & $57.1 \%$ & 0.360 \\
\hline Women & $36.0 \%$ & $42.9 \%$ & \\
\hline Mean age, years & 62.3 & 64.07 & 0.566 \\
\hline $\begin{array}{l}\text { Mean time since } \\
\text { ALS onset, months }\end{array}$ & 17.96 & 17.98 & 0.879 \\
\hline Lower education ( $<8$ years) & $48 \%$ & $35.7 \%$ & 0.209 \\
\hline Higher education & $52 \%$ & $64.3 \%$ & \\
\hline Normal & $36.0 \%$ & $28.6 \%$ & 0.341 \\
\hline Some cognitive impairment & $64.0 \%$ & $71.4 \%$ & \\
\hline No dementia & $88 \%$ & $96.4 \%$ & 0.206 \\
\hline Dementia & $12 \%$ & $3.6 \%$ & \\
\hline Mean ALS-CBS & 12 & 12.11 & 0.909 \\
\hline Mean ALSFRS-R score & 29.16 & 26.7 & 0.272 \\
\hline Mean FVC & $79 \%$ & $69 \%$ & 0.125 \\
\hline Bulbar onset & $22 \%$ & $17.9 \%$ & 0.457 \\
\hline $\begin{array}{l}\text { Bulbar signs at the time } \\
\text { of cognitive assessment }\end{array}$ & $58 \%$ & $53.6 \%$ & 0.443 \\
\hline
\end{tabular}

ALS: amyotrophic lateral sclerosis; ALS-CBS: amyotrophic lateral sclerosis cognitive behavioural screen; ALSFRS-R: revised amyotrophic lateral sclerosis functional rating scale; FCV: forced vital capacity. with any impairment (ALSci, ALSbi, ALScibi, ALS-FTD) and the second included patients without ALS-FTD (normal, ALSci, ALSbi, ALScibi) versus ALS-FTD patients. The sensitivity, specificity, positive predictive value, and negative predictive value of the ALS-CBS in the two scenarios are shown in Table III with the respective cut-offs.

In the first scenario (ALS normal vs. ALS any impairment), the following variables showed significant differences: mean age (56.7 vs. 65.4 years, $p<0.014)$, low educational level $(<8$ years schooling, $16.7 \%$ vs. $65.6 \% ; p<0.001)$, percentage with depression ( $5.6 \%$ vs. $34.4 \%, p<0.036)$, ALS-CBS cognitive score $\leq 15$ (33.3\% vs. $81.8 \%, p<0.0001)$, ALS-CBS behavioural score $\leq 36(22.2 \%$ vs. $59.4 \%$, $p<0.018)$, mean ALS-CBS cognitive and behavioural scores ( 16.6 vs. $9.66, p<0.0001$ and 40.11 vs. $30.41, p<0.001$, respectively), and presence of a palmomental reflex $(55.6 \%$ vs. $84.4 \%, p<0.03)$. There were no significant differences in gender, disease duration, or type of onset (spinal vs. bulbar).

Differences in the type of onset were found between patients with cognitive impairment: $34.5 \%$ of patients with cognitive involvement (ALSci, ALScibi, FTD) had a bulbar onset, whereas only $4.8 \%$ of patients without cognitive deterioration debuted in this manner $(p<0.016)$.

On logistic regression analysis, independent variables related to a risk of developing cognitive impairment were age $(\operatorname{Exp}(\mathrm{B}): \quad 1.079$; CI 1.031-1.130) and ALS-CBS cognitive score $(\operatorname{Exp}(B): 0.731 ;$ CI 0.603-0.886).

In the second scenario (ALS with FTD vs. ALS without FTD), significant differences were seen for bulbar onset ( $66.7 \%$ vs. $15.9 \%, p<0.017)$, development of bulbar symptoms $(100 \%$ vs. $52.3 \%$, $p<0.033)$, ALS-CBS cognitive score $\leq 8$ ( $83.3 \%$ vs. $25.0 \%, p<0.01)$, ALS-CBS behavioural score $\leq 35$ ( $83.3 \%$ vs. $34.1 \%, p<0.032$ ), and mean ALS-CBS cognitive and behavioural scores (5.3 vs. 12.8 , $p<0.0001$ and 24.17 vs. $35.2, p<0.027$, respectively). We found no significant differences in gender,

Table III. Neurological diagnoses and neuropsychological diagnoses. Sensitivity, specificity, positive predictive value (PPV), negative predictive value (NPV) and area under the curve (AUC) of the ALS-CBS validated in our ALS population.

\begin{tabular}{llllll}
\hline & Normal, $\%$ & ALSci, $\%$ & ALSbi, $\%$ & ALScibi, $\%$ & ALS-FTD, $\%$ \\
\hline Neurological diag. & 30 & 34 & 8 & 16 & 12 \\
Neuropsychological diag. & 36 & 34 & 6 & 12 & 12 \\
& Sensitivity, $\%$ & Specificity, $\%$ & PPV, \% & NPV, $\%$ & Kappa \\
& 100 & 83.3 & 91.4 & 100 & 0.85 \\
Neurologist vs. Neuropsychologist, to classify Normal vs. CI & Sensitivity, $\%$ & Specificity, $\%$ & PPV, $\%$ & NPV, $\%$ & AUC, $\%$ \\
& 83.3 & 75 & 31.3 & 97.1 & 87.3 \\
ALS-CBScog, cut-off: 8 No FTD vs. FTD & 86.2 & 62 & 75.8 & 76.5 & 84.7 \\
ALS-CBScog, cut-off: 15 Normal vs. CI & 83.3 & 69 & 25 & 96.7 & 80.9 \\
ALS-CBSbv, cut-off: 35 No FTD vs. FTD & 93.3 & 74.3 & 61 & 96.3 & 83.3 \\
ALS-CBSbv, cut-off: 36 Normal vs. CI & & & & &
\end{tabular}

ALSci: amyotrophic lateral sclerosis with cognitive impairment; ALSbi: amyotrophic lateral sclerosis with behavioural impairment; ALScibi: amyotrophic lateral sclerosis with cognitive and behavioural impairment; ALS-FTD: amyotrophic lateral sclerosis with frontotemporal dementia; diag: diagnoses; CI: cognitive impairment; ALS-CBScog: ALS-CBS cognitive subscale; FTD: frontotemporal dementia; ALS-CBSbv: ALS-CBS behavioural subscale. 
age, educational level, presence of depression or palmomental reflex, or disease duration.

On logistic regression analysis, the ALS-CBS cognitive score was independently related to FTD $(\operatorname{Exp}(\mathrm{B}): 0.793 ; \mathrm{CI}$ 0.707-0.891). In both logistic regression analyses, we used the diagnoses based on the neuropsychological assessment.

There were no significant differences in the mean FVC values or ALSFRS-R scores between the groups in either of the two scenarios analysed.

\section{Discussion}

Woolley et al. developed the ALS-CBS as a quick screening instrument to detect cognitive/behavioural impairment in ALS. In the present study, a version of this instrument in the Spanish language was developed and validated on a sample of ALS patients in our setting. The Spanish ALS-CBS proved useful for screening in this population. The sensitivity and specificity values were similar to those of the original instrument (5), although optimal cut-off scores for screening were lower, probably because of the relatively low educational level of our population. As occurred with the original instrument, the performance of the behavioural section was somewhat lower than the cognitive section. We obtained excellent negative predictive values that enabled acceptably reliable estimation of which patients did not present impairment or dementia. The lower positive predictive values imply that a higher percentage of patients will require extensive neuropsychological evaluation.

The utility of the clinical-neurological evaluation (including ALS-CBS) approached that of the reference neuropsychological assessment for determining whether patients had cognitive/behavioural impairment. In regular practice there would be a considerable time saving for the system and the patient if only the clinical-neurological evaluation were performed in the initial assessment. Based on this simplified screening, patients with cognitive impairment could then be reliably referred to a specialist for further evaluation. Neuropsychological assessment has a major impact on the patient, as is evidenced by the large percentage of our initial cohort who could not complete or refused to complete the evaluation (35\%).

The percentages of patients placed in the various cognitive impairment diagnostic groups were similar to reported values $(1,28,29)$. The high percentage with cognitive impairment and the rate with which this condition is underdiagnosed were similar to values reported in an epidemiological study performed in Barcelona (80\%) (30). The clinicalneurological evaluation coincided with the reference standard evaluation in most cases of cognitive impairment, with full concordance for FTD. In keeping with reported findings, the most highly affected cognitive domain in our patients was executive function $(1,28,29)$, although a recent study has cited language as the most profoundly affected domain (31). To further investigate this aspect, we reanalysed the affected cognitive domains excluding verbal fluency from executive function; nonetheless, executive function persisted as the domain showing the greatest changes. One potential explanation for the differences between studies of this type may be the design of the neuropsychological batteries used. A greater presence of tests examining a specific domain may increase the sensitivity for detecting changes in that area.

As has been described $(1,32)$, a higher percentage of ALS patients with bulbar onset had FTD than those with spinal onset. This difference was not found in the analysis of patients with no impairment versus any impairment, but it was when patients with pure behavioural disorder were excluded.

As this is not a population based study, we are unable to provide definite conclusions regarding the contribution of traditional risk factors such as age and educational level on the risk of experiencing FTD or mild impairment. Nonetheless, our findings concur with those of Woolley, in that educational level is not a determinant of risk in these patients; it is a risk factor for Alzheimer's disease and for dementia in general $(30,33)$, but not for FTD (34). The initial significance of this factor in our analysis may be related to the role of age as an independent variable associated with cognitive impairment. In our specific population, the older the age, the greater the likelihood that patients would have a lower educational level. Age seemed to be an independent risk factor for the diagnosis of impairment in general, but not specifically for FTD, perhaps because of the low number of FTD patients included in the analysis. An increase in the incidence of FTD has been described with ageing, up to the age of 70 years (34). In our series, $65 \%$ of patients were younger than 70 years of age, and this may be why we found a certain age-associated risk for presenting any type of cognitive/behavioural impairment.

The role of depression as a confounding factor remains to be resolved $(1,5)$. Depression could be a psychobehavioural symptom associated with cognitive impairment in ALS or an adaptive state that interferes with the patients' cognitive performance. In our series, $24 \%$ of patients presented depressive symptoms on evaluation, mild in $90 \%$. Nonetheless, the data do not suggest overdiagnosis of FTD due to depression, since the percentage with this factor was similar between those with and without FTD. Furthermore, although depression was more common in patients with any impairment than in those without, it was not an independent risk factor for impairment on multivariate analysis $(p=0.1)$. An elevated rate of depression has been reported in caregivers of ALS patients, and this has generated 
interest in studying the impact of the disease on quality of life of the patients' family members $(35,36)$. Better knowledge of the patients' cognitive and behavioural situation would favour optimal management by physicians and improvements for caregivers, who could experience reductions in their care burden.

Another issue that must be addressed is whether cognitive impairment or behavioural disorder are independently related to a poor prognosis in these patients or are actually self-fulfilling prophesies, being linked with the use of less intensive therapy. It could be in the patient's interest to establish clear lines of action regarding therapy. For example, future decisions could be protocoled early, when incipient impairment is first detected. It could also be helpful to create instruments to determine the capacity of a patient with impairment or FTD to take decisions. According to some authors, behavioural disorder (37) and executive function changes $(38,39)$ are associated with a poorer prognosis, whereas others have not found these relationships (40). The future follow-up of our patients will enable us to discuss this issue.

In contrast to Woolley's results (5), we found no correlation between ALS-CBS scores and FVC values or ALSFRS scores $(p>0.05)$. Nor did we detect significant differences in the mean FVC or ALSFRS between patients with and without any impairment, or between those with and without dementia. Hence, we concur with Woolley's suggestion that this association would result from the influence of the bulbar-onset form for both factors. It seems equally reasonable that there would be no relationship with the functional status (ALSFRS), since FTD and ALS can be onset forms or appear sequentially in an interchangeable manner.

\section{Conclusions}

In conclusion, the Spanish version of the ALS-CBS test is a useful instrument for screening cognitive/ behavioural impairment in ALS patients in our setting. We found a high rate of impairment in the cohort evaluated, especially in those with a bulbar onset; hence, it seems advisable to include cognitive screening in the initial evaluation of these patients. This practice could result in better patient management, benefits for caregivers, and improved decision-making.

\section{Acknowledgements}

This study was supported by Fundación Mutua Madrileña. The authors are grateful to Susan Woolley for graciously granting us use of the ALSCBS, and to her team for their help. We thank Fundació Miquel Valls, our appreciate team and, particularly, all the participating patients.
References 39 and 40 refer to supplementary material, available via the supplementary tab on the article's online page at http://dx.doi.org/10.3109/ 21678421.2015.1125500

Declaration of interest: The authors declare no conflicts of interest.

\section{References}

1. Achi EY, Rudnicki SA. ALS and Frontotemporal Dysfunction: A Review. Neurol Res Int. 2012;2012:806306.

2. Neary D, Snowden JS, Gustafson L, Passant U, Stuss D, Black S, et al. Frontotemporal lobar degeneration: a consensus on clinical diagnostic criteria. Neurology. 1998;51:1546-54.

3. Petersen RC, Negash S. Mild cognitive impairment: an overview. CNS Spectr. 2008;13:45-53.

4. Strong MJ, Grace GM, Freedman M, Lomen-Hoerth C, Woolley S, Goldstein LH, et al. Consensus criteria for the diagnosis of frontotemporal cognitive and behavioural syndromes in amyotrophic lateral sclerosis. Amyotroph Lateral Scler. 2009; 10:131-46.

5. Woolley SC, York MK, Moore DH, Strutt AM, Murphy J, Schulz PE, Katz JS. Detecting frontotemporal dysfunction in ALS: utility of the ALS Cognitive Behavioural Screen (ALSCBS). Amyotroph Lateral Scler. 2010;11:303-11.

6. Brooks BR, Miller RG, Swash M, Munsat TL. World Federation of Neurology Research Group on Motor Neuron Diseases. El Escorial revisited: revised criteria for the diagnosis of amyotrophic lateral sclerosis. Amyotroph Lateral Scler Other Motor Neuron Disord. 2000;1:293-9.

7. Cedarbaum JM, Stambler N, Errol M, Fuller C, Hilt D, Thurmond B, et al. The ALSFRS-R: a revised functional rating scale that incorporates assessments of respiratory function. J Neurol Sci. 1999;169:13-21.

8. Folstein MF, Folstein SE, McHugh PR. 'Mini-Mental State'. A practical method for grading the cognitive state of patients for the clinician. J Psychiat Res. 1975;12:189-98.

9. Peña-Casanova J. Programa Integrado de Exploración Neuropsicológica. Test Barcelona. Peña-Casanova, Masson, editors. Barcelona 1991.

10. Artiola L, Hermosillo D, Heaton R, Pardee RE. Manual de normas y procedimientos para la batería neuropsicológica en español. mPress. Tucson, AZ 1999.

11. Stroop JR. Studies of interference in serial verbal reaction. Journal of Experimental Psychology. 1935;18:643-62.

12. Partington J, Leiter R. Partington's pathways test. The Psychological Service Centre Bulletin. 1949;1:9-20.

13. Heaton RK. WCST: Test de Clasificación de Tarjetas de Wisconsin (adaptación española, 3rd edn.). TEA Ediciones, S.A. Madrid 2001.

14. Culbertson W, Zillmer EA. TOL- DX Tower of London Drexel University. Multi-Health Systems. Chicago 2001.

15. Wechsler D. WAIS-III: Escala de Inteligencia de Wechsler para adultos III (adaptación española ed.). TEA Ediciones, S.A. Madrid 1999.

16. Peña-Casanova J. Programa Integrado de Exploración Neuropsicológica: Test Barcelona Revisado. TBR (2nd edn.). Masson, S.A. Barcelona 2005.

17. Quiñones-Ubeda S, Peña-Casanova J, Böhm P, GramuntFombuena N, Comas L. Estudio normativo piloto de la segunda edición del Boston Naming Test en una muestra española de adultos jóvenes (20 a 49 años). Neurología. 2004;19: 248-53.

18. Gudayol-Ferré E, Lara JP, Herrera-Guzman I, Böhm P, Rodés E, Ansaldo AI, et al. Semantic memory as assessed by the Pyramids and Palm Trees Test: the impact of 
sociodemographic factors in a Spanish-speaking population. J IntNeuropsychol Soc. 2008;14:148-51.

19. Rey A. El examen clínico en psicología. Ed: Kpelusz. Buenos Aires 1962.

20. Wechsler D. WMS-III: Escala de Memoria de Wechsler III. Ed: Departamento I + D Madrid, TEA ediciones, S.A. Madrid 2004.

21. Benton AL, Hannay HJ, Varney N. Visual perception of line direction in patients with unilateral brain disease. Neurology. 1975;25:907-10.

22. Peña-Casanova J, Blesa R, Aguilar M, Gramunt-Fombuena N, Gómez-Ansón B, Oliva R, et al. NEURONORMA Study Team. Spanish Multicentre Normative Studies (NEURONORMA Project): methods and sample characteristics. Archives of Clinical Neuropsychology. 2009;24:307-19.

23. Beck A, Steer R, Brown G. Inventario de Depresión de Beck-II. Ed: Paidós. Buenos Aires 2006.

24. Cummings JL, Mega M, Gray K, Rosenberg-Thompson S, Carusi D, Gornbein J. The neuropsychiatric inventory. Comprehensive assessment of psychopathology in dementia. Neurology. 1994;44:2308-14.

25. Vilalta J, Lozano M, Hernández M, Llinàs-Reglà J, LópezPousa S, López O. Neuropsychiatric Inventory. Propiedades psicométricas de su adaptación al español. Revista de Neurología. 1999;29:15-19.

26. Marin RS. Differential diagnosis and classification of apathy. Am J Psychiatry. 1990;147:22-30.

27. Kertesz A, Davidson W, Fox H. Frontal behavioural inventory: diagnostic criteria for frontal lobe dementia. Canadian Journal of Neurological Sciences. 1997;24:29-36.

28. Ringholz GM, Appel SH, Bradshaw M, Cooke NA, Mosnik DM, Schulz PE. Prevalence and patterns of cognitive impairment in sporadic ALS. Neurology. 2005;65:586-90.

29. Phukan J, Elamin M, Bede P, Jordan N, Gallagher L, Byrne $\mathrm{S}$, et al. The syndrome of cognitive impairment in amyotrophic lateral sclerosis: a population based study. J Neurol Neurosurg Psychiatry. 2012;83:102-8.

30. Gascón-Bayarri J, Reñé R, del Barrio JL, de Pedro-Cuesta J, Ramón JM, Manubens JM, et al. Prevalence of dementia subtypes in El Prat de Llobregat, Catalonia, Spain: the PRATICON study. Neuroepidemiology. 2007;28:224-34.
31. Taylor LJ, Brown RG, Tsermentseli S, Al-Chalabi A, Shaw $\mathrm{CE}$, Ellis CM, et al. Is language impairment more common than executive dysfunction in amyotrophic lateral sclerosis? J Neurol Neurosurg Psychiatry. 2013;84:494-8.

32. Lillo P, Garcin B, Hornberger M, Bak TH, Hodges JR. Neurobehavioural features in frontotemporal dementia with amyotrophic lateral sclerosis. Arch Neurol. 2010;67:826-30.

33. López-Pousa S. Definición, prevalencia, incidencia y factores de riesgo de la enfermedad de Alzheimer. In Enfermedad de Alzheimer y otras Demencias, 4th edn. Alberca R, LópezPousa S, editors. Editorial Médica Panamericana. Madrid 2011.

34. Ojea T. Clínica y tratamiento de la variante conductual de la demencia frontotemporal. In Enfermedad de Alzheimer y otras Demencias 4th edn. Alberca R, López-Pousa S, editors. Editorial Médica Panamericana. Madrid 2011.

35. Lillo P, Mioshi E, Hodges JR. Caregiver burden in amyotrophic lateral sclerosis is more dependent on patients' behavioural changes than physical disability: a comparative study. BMC Neurol. 2012;12:156.

36. Chiò A, Vignola A, Mastro $\mathrm{E}$, Giudici $\mathrm{AD}$, Iazzolino $\mathrm{B}$, Calvo A, et al. Neurobehavioural symptoms in ALS are negatively related to caregivers' burden and quality of life. Eur J Neurol. 2010;17:1298-303.

37. Phukan J, Pender NP, Hardiman O. Cognitive impairment in Amyotrophic Lateral Sclerosis. Lancet Neurol. 2007;6:9941003.

38. Gordon PH, Goetz RR, Rabkin JG, Dalton K, McElhiney $\mathrm{M}$, Hays AP, et al. A prospective cohort study of neuropsychological test performance in ALS. Amyotroph Lateral Scler. 2010;11:312-20.

39. Elamin M, Bede P, Byrne S, Jordan N, Gallagher L, Wynne $\mathrm{B}$, et al. Cognitive changes predict functional decline in ALS: a population based longitudinal study. Neurology. 2013;80:1590-7.

40. Rusina R, Ridzon P, Kulisták P, Keller O, Bartos A, Buncová $\mathrm{M}$, et al. Relationship between ALS and the degree of cognitive impairment, markers of neurodegeneration and predictors for poor outcome. A prospective study. Eur J Neurol. 2010;17:23-30. 